\title{
Éditorial
}

\section{Analyse territoriale en ressourcement}

Lors d'un important colloque tenu en novembre 2003 à Philadelphie, la Regional Science Association International célébrait son $50^{\mathrm{e}}$ anniversaire. Ce fut un moment solennel et surtout émouvant pour tous ces professeurs, chercheurs, étudiants et experts de l'analyse régionale qui, précisons-le, ont vécu au début des années 1970 un questionnement profond sur la pertinence sociale de cette discipline scientifique. Le nombre et la qualité des communications livrées à ce colloque anniversaire confirment que les territoires urbains, ruraux, régionaux, métropolitains et périphériques, qui composent les nations, demeurent un objet scientifique de grande préoccupation en cette ère de mondialisation. D’une manière plus générale dans la littérature scientifique récente, il apparaît que les contributions sur les quatre principaux concepts de l'analyse territoriale obtiennent un regain d'intérêt. Regain scientifi- que qui peut avantageusement éclairer la pratique territoriale au Québec. Voyons un peu.

La cité et son pendant, la théorie urbaine, obtiennent actuellement de très nombreuses contributions scientifiques par l'entremise d'un vaste mouvement de recherche largement stimulé par le phénomène de la «métropolisation». Même si les approches «citésjardins », « urban planning », «économie d'agglomération » et «technopoles 》 demeurent toujours très pertinentes, un avancements significatif de nos connaissances cumulées se situe actuellement sous l'angle des « réseaux urbains». En contexte québécois, l'une des principales questions que l'on peut poser à ce corpus théorique en plein développement concerne le « comment connecter davantage nos villes petites, moyennes et plus grandes, dispersées et distantes sur un très vaste espace, dans les réseaux renouvelés de l'économie continentale et mondiale ».

\section{Les quatre principaux concepts de l'analyse territoriale}

\begin{tabular}{l|r} 
La Cité & La Région \\
\hline La Communauté & Le District
\end{tabular}

Le concept de communauté en analyse territoriale bénéficie aussi de nombreuses contributions scientifiques qui participent à son renouvellement. Deux échelles communautaires s'avèrent particulièrement vivaces à cet effet dans la littérature scientifique, soit le supralocal (intermunicipal) et le quartier dans les villes. Plusieurs questions sont investiguées par les chercheurs, notamment celle concernant le diptyque « coopération - concurrence ». Bernard Pecqueur, de Grenoble, qui sera au mois de mai le conférencier d'ouverture du colloque de la section Développement régional de l'ACFAS 2004, est justement un répon- dant à cette question qui devient très pertinente au Québec. Autant les territoires MRC que les nouveaux arrondissements des agglomérations urbaines se questionnent en effet sur le «comment bonifier l'esprit communautaire », en particulier par l'entremise de la décentralisation, de l'appropriation, de la solidarité et de la gouvernance. La recherche sur le développement local apporte plusieurs réponses en ce sens.

Le régionalisme n'est pas en reste dans le renouvellement actuel de l'analyse territoriale. Furent encore récemment proposés à la recherche plusieurs concepts 
aussi stimulants que fertiles tels que l'État-région, la "Global City-Region», la région qui gagne, la « learning region ». Ces concepts servent bien l'enjeu d'une meilleure compréhension et maîtrise de la stratégie typiquement régionale au travers des autres stratégies de développement et de gestion. En considérant la turbulente mais néanmoins fructueuse construction institutionnelle des régions administratives qui a caractérisé au Québec les années 1970 et 1980, nul doute que la théorie régionale en renouvellement pourrait être inspirante, sinon rafraîchissante, pour les acteurs régionaux devenus de trop sages stratèges. On pourrait y tirer certains enseignements concrets à propos du rôle de la prospective, du partenariat publicprivé, du renouvellement des décideurs, de la culture organisationnelle, des coalitions, etc. pour dynamiser un régionalisme québécois qui semble en avoir bien besoin.

Le quatrième concept important de l'analyse territoriale représente celui qui, comparativement, a obtenu le plus d'avancement scientifique au cours des deux dernières décennies en enrichissant considérablement son corpus théorique déjà ancien. La modélisation des nouveaux districts italiens (NDI) a fait émerger, en effet, un vaste courant de recherche empirique. Son vocabulaire est très riche, fertilisé notamment par la théorie du développement endogène. Tant et si bien que les « clusters », créneaux territoriaux, milieux innovateurs, niches incubatrices, systèmes territoriaux de production sont devenus l'objet d'un très vif intérêt pour la politique territoriale autant en Amérique qu'en Asie, mais surtout en Europe. Divers enjeux sont soulevés à cet effet dans la littérature, notamment les innovations institutionnelles capables de tisser des relations de collaboration entre les divers acteurs du développement de ces filières de production encastrées sur le territoire où elles siéent. Le Québec doit tirer profit de la richesse analytique de cette approche " district» tout à fait pertinente pour l'intervention. D'autant plus que les filières de production (forêt, multimédia, bleuets, bio-alimentaire, aérospatial, textiles techniques, produits de la mer, tourbes...), encore peu développées généralement, illustrent une grande variété de territorialités qui ne correspondent que rarement à des découpages reconnus officiellement par les autorités publiques. Dans le contexte institutionnel actuel, comment soutenir davantage la structuration des filières territoriales de production à la québécoise ?

Ce numéro $1 \mathrm{du}$ volume 13 ne traite évidemment pas de toutes ces questions et de tous ces apports scientifiques. Mais nous les traitons sur une longue période et ce, toujours dans un esprit de vulgarisation scientifique. Je vous souhaite une bonne lecture des textes sélectionnés pour vous.

Marc-Urbain Proulx Université du Québec à Chicoutimi 